\title{
Tongue granuloma: An unusual complication of potassium permanganate ingestion
}

\author{
E Motloung, MMed, FCPaed Surg; N Mashaba, MB ChB \\ Department of Paediatric Surgery, Mankweng Tertiary and Academic Hospital, University of Limpopo, Polokwane, South Africa
}

Corresponding author: E Motloung (drmotloung@me.com)

Potassium permanganate $\left(\mathrm{KMnO}_{4}\right)$ is a powerful oxidising agent. In its raw form, it is an odourless, sweet-tasting, dark-purple substance, available in ready-to-use solutions, pellets, tablets, crystals and powder form. In clinical practice historically, it has been used as an antiseptic and antifungal agent. In rural South African communities, however, $\mathrm{KMnO}_{4}$ has many traditional uses, including use as a snake repellent, a treatment for constipation, joint pains, open wounds, urticaria and even for casting out spells. $\mathrm{KMnO}_{4}$ is kept in most households in rural Limpopo Province, and is therefore easily accessible by children, and alluring because of its sweet taste and similarity to candy. The corrosive effects of $\mathrm{KMnO}_{4}$ ingestion on the gastrointestinal tract are secondary to the formation of potassium hydroxide, a strong alkaline corrosive which causes liquefactive necrosis, allowing deep penetration into mucosal tissue as cells are destroyed. The most common adverse effect of $\mathrm{KMnO}_{4}$ ingestion observed in our practice, and reported in this case report, is an oesophageal stricture. This adverse event often results in children dying from severe gastrointestinal bleeds or complications of tracheoesophageal fistula associated with upper airway obstruction.

S Afr Med J 2021;111(11b):1149-1150. https://doi.org/10.7196/SAMJ.2021.v111i11b.16129

Potassium permanganate $\left(\mathrm{KMnO}_{4}\right)$ is a powerful oxidising agent. In its raw form, it is an odourless, sweet-tasting and dark purple substance, available in ready-to-use solutions, pellets, tablets, crystals and powder form. ${ }^{[1]}$ It is readily accessible to our rural population over the counter at most local stores and without prescription at pharmacies. In clinical practice, historically it has been used as an antiseptic and antifungal agent. In rural South African (SA) communities, $\mathrm{KMnO}_{4}$ has many traditional uses, including: use as a snake repellent, by mixing it in water and sprinkling it around the yard; treating constipation, joint pains, open wounds, and urticaria; and even for casting out spells. ${ }^{[1]}$ The modes of administration differ, as they involve bathing in the solution, oral ingestion, enema administration and herbal compresses. $\mathrm{KMnO}_{4}$ is kept in most households in our population for such uses, and is therefore easily accessible by children, and alluring because of its sweet taste and similarity to candy. ${ }^{[1]}$

Symptoms and complications of $\mathrm{KMnO}_{4}$ ingestion in children have been documented, and include dysphagia, odynophagia, nausea and vomiting, the result of gastrointestinal oedema, erosion and ulcerations of the gastrointestinal epithelium. ${ }^{[2]}$ Severe complications include oesophageal perforation, mediastinitis associated with upper airway obstruction, hepatic and renal toxicity, and cardiovascular depression. ${ }^{[2]}$ The most common adverse effect of $\mathrm{KMnO}_{4}$ ingestion observed in our practice, and reported in this case report, is oesophageal stricture. Many patients have died from severe gastrointestinal bleeds and tracheoesophageal fistula associated with upper airway obstruction. ${ }^{[2]}$ The corrosive effects of $\mathrm{KMnO}_{4}$ ingestion on the gastrointestinal tract is secondary to the formation of potassium hydroxide, a strong alkaline corrosive which causes liquefactive necrosis, allowing deep penetration into mucosal tissue as cells are destroyed. ${ }^{[2]}$ Its over-the-counter availability has contributed to its harmful potential being overlooked. It is reported that children ingest $\mathrm{KMnO}_{4}$ after mistaking it for sweets or juice. ${ }^{[1]}$

\section{Case}

A 3-year-old girl presented to casualty after ingestion of $\mathrm{KMnO}_{4}$ crystals, following which she had episodes of vomiting and difficulty in breathing. On arrival, she was conscious but irritable, crying, with tachypnoea and stridor. Her vitals were as follows: heart rate $140 \mathrm{bpm}$, respiratory rate 29 breaths per minute, and oxygen saturation $\left(\mathrm{SpO}_{2}\right)$ was $74 \%$ on room air. On physical examination, her oral cavity showed a very swollen tongue, with brown stains on the tongue and teeth. Her airway was obstructed by the swollen tongue and stridor was present. Her breathing was rapid. Systemic examination was unremarkable. All her laboratory values were normal. Chest radiograph was normal. Her past medical history was unremarkable. She was intubated and admitted to the intensive care unit (ICU) for 3 days. Our service was consulted 3 days after ICU admission and extubation. As this was 72 hours post trauma, endoscopy could not be done.

After extubation, the patient had hypersalivation and was not tolerating oral feeds. She was given total parenteral nutrition (TPN). A barium swallow on day 14 revealed irregularity and narrowing of the proximal oesophagus, with no evidence of perforation.

She underwent endoscopy and was found to have a stricture $10 \mathrm{~cm}$ into the proximal oesophagus. Balloon dilatations were performed to a pressure of only $2 \mathrm{mmHg}$. A decision was made to place a gastrostomy tube.

Three weeks post trauma she presented with a mass on the top of the tongue which was pulling the tip of the tongue superiorly and posteriorly (Fig. 1). On examination, an assessment of a tongue granuloma as a complication of caustic ingestion was made. Complete excision was done in theatre (Fig. 2).

Follow-up endoscopies were attempted but full dilation was still not possible because of the stricture being tight; the only option left was surgical management. The patient was then taken for a resection of the affected stricture and anastomosis. A barium swallow was done on day 5 post surgery (Fig. 3) which showed no leak of contrast and she was discharged, with a follow-up appointment in 2 weeks.

Two weeks after surgery she had saliva leaking from the cervical incision, which was evidence of anastomotic breakdown.

\section{Discussion}

Caustic and corrosive substance ingestion causes tissue injury by 


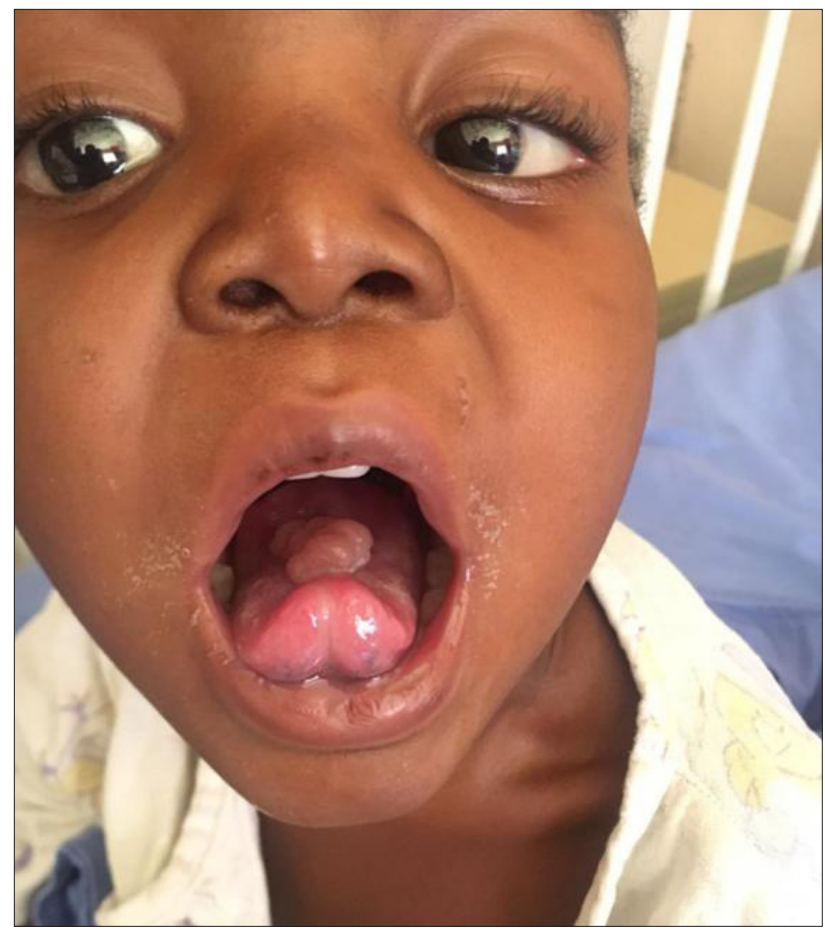

Fig. 1. Granuloma pulling the tip of the tongue superiorly and posteriorly.

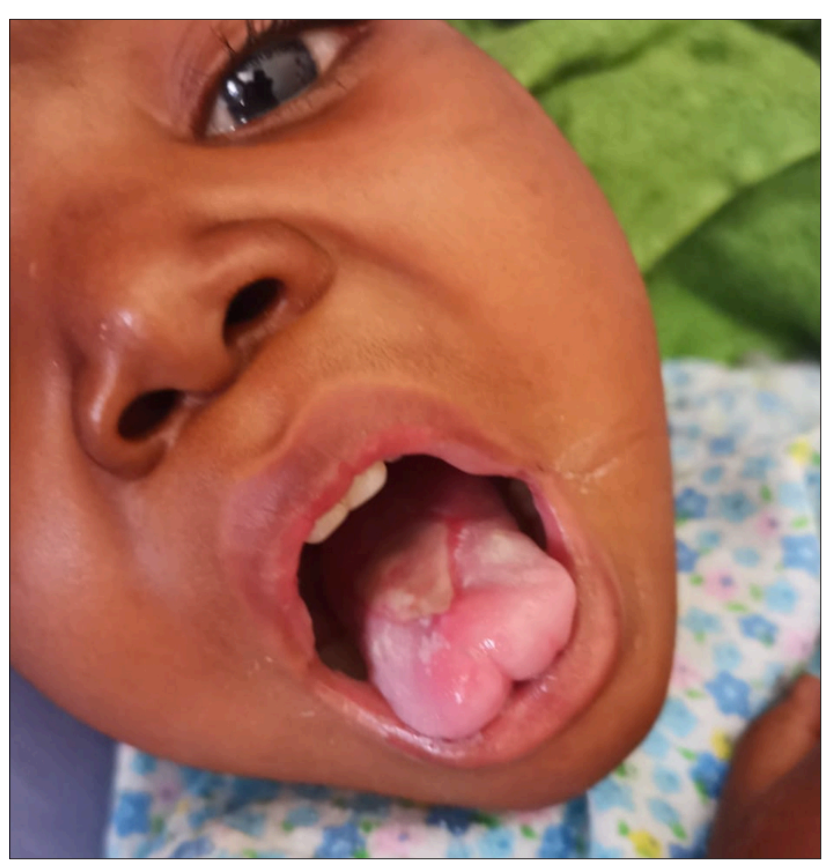

Fig. 2. Post excision of the granuloma.

chemical reaction. ${ }^{[3]}$ Both acidic and alkaline substances are involved (Table 1). ${ }^{[2]}$ Ingestion of these substances can be deliberate, as in suicide in adults, or accidental, in children under 6 years old. ${ }^{[4]}$ The outcome of ingesting these substances can be devastating and depends on several factors, e.g. the physical form of the ingested product, type, amount, concentration, pre-ingestion condition of tissues and duration of contact. ${ }^{[2]}$ Commonly ingested substances in $\mathrm{SA}$, including domestic cleaning products, with their $\mathrm{pH}$ values, are listed in Table 2.

Strong acids are the most commonly ingested corrosive chemicals. They cause immediate severe oropharyngeal pain. This may be the reason they are usually consumed in small volumes compared with

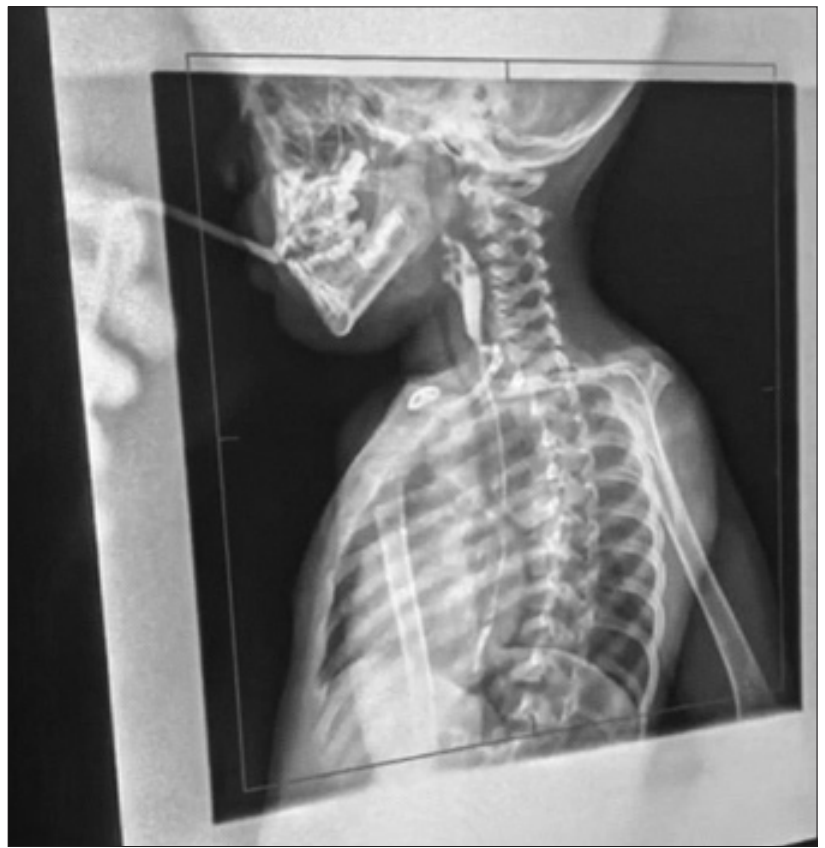

Fig. 3. Contrast swallow 5 days after resection of oesophageal stricture and anastomosis.

\begin{tabular}{ll}
\hline \multicolumn{2}{l}{ Table 1. Acid and alkaline substances } \\
\hline Type & Example \\
\hline Alkali & $\begin{array}{l}\text { Sodium hydroxide, potassium hydroxide, (oven } \\
\text { cleaners, liquid agents, liquid drain cleaners, } \\
\text { disc batteries), calcium and lithium hydroxide } \\
\text { (hair relaxers), ammonia (household cleaners), } \\
\text { dishwasher detergents } \\
\text { Sulphuric acid, hydrochloric acid, nitric acid } \\
\text { (toilet bowel cleaners, swimming pool cleaners, } \\
\text { rust removers) }\end{array}$ \\
Acid & $\begin{array}{l}\text { Hypochlorous acid (bleach - generally neutral } \\
\text { pH commercially), peroxide (mildew remover) }\end{array}$ \\
\end{tabular}

\begin{tabular}{ll} 
Table 2. Commonly ingested acid and alkaline substances \\
\hline $\mathbf{p H}$ & Examples of solutions \\
\hline 0 & Battery acid, strong hydrofluoric acid \\
1 & Hydrochloric acid secreted by stomach lining \\
2 & Lemon juice, gastric acid, vinegar \\
3 & Grapefruit juice, orange juice, soda \\
4 & Tomato juice, acid rain \\
5 & Soft drinking water, black coffee \\
6 & Urine and saliva \\
7 & Pure water \\
8 & Sea water \\
9 & Baking soda \\
10 & Great Salt Lake, milk of magnesia \\
11 & Ammonia solution \\
12 & Soapy water \\
13 & Bleach, oven cleaner \\
14 & Liquid drain cleaner \\
\end{tabular}

alkaline substances. They have less surface tension and pass rapidly through the oesophagus, resulting in fewer oesophageal injuries. ${ }^{[2]}$ They most commonly cause gastric injuries. 
Strong alkaline substances produce deep injuries by adhering to the mucosa of the mouth and oesophagus, mostly sparing the stomach. ${ }^{[2]}$ They combine with tissue proteins to cause liquefactive necrosis and saponification, leading to full-thickness damage and vascular thrombosis. Granular products cause more serious injuries owing to prolonged-contact mucosal time. ${ }^{[2]}$ Ingestion of alkaline disc batteries containing $45 \%$ solution of potassium hydroxide or sodium hydroxide leads to leakage and damage to the oesophagus within 1 hour and perforation in $8-12$ hours. Emergency removal is mandatory. ${ }^{[2]}$

Following caustic ingestion, patients may be asymptomatic or present with nausea, vomiting, dysphagia, odynophagia, drooling, abdominal pains, respiratory distress and stridor.

Initial management includes airway management, fluid resuscitation and stabilisation. Assessment of oesophageal/gastric injury through flexible upper endoscopy should be done within 24 48 hours of ingestion, unless there is evidence of perforation, which can be seen on chest and abdominal X-rays. The injured oesophagus is weakest between day 7 and day 21 post ingestion; ${ }^{[2]}$ therefore endoscopy should not be performed later than 5 days post ingestion to minimise risk of bleeding and perforation. ${ }^{[2]}$

Patients with no symptoms, no oral lesions and vague history do not have to undergo endoscopy. They can be given oral fluids and go home after being observed for a few hours. ${ }^{[2]}$ Classification of caustic injuries is similar to that of burns. This classification is based on endoscopic evaluation and is used to predict clinical outcome and course. ${ }^{[5]}$

Grade 0 - 2a injuries can take oral fluids and be discharged once symptoms resolve. Grade $2 \mathrm{~b}-3$ injuries should be observed for 24 48 hours before starting feeds. ${ }^{[2]}$ Proton pump inhibitors, antibiotics and antifungal agents are given for injuries classified as grade $2 \mathrm{~b}$ and above. Oesophageal strictures are common in severe injuries. They occur in $70 \%$ of patients with grade $2 \mathrm{~b}$ and in $100 \%$ of patients with grade 3 injuries. Patients with grade $2 b-3$ injuries should have a barium swallow 2 - 3 weeks after injury to check for a possible stricture. ${ }^{[2]}$

After a stricture has been identified, the cornerstone of nonoperative treatment is dilatations performed with a balloon dilator or semi-rigid Savary-Gilliard bougies (Cook Medical, USA). When non-operative management fails, operative management becomes necessary. Currently accepted surgical management includes resection of the affected segment with end-to-end anastomosis or oesophageal replacement.

\section{Conclusion}

Easy access to a dangerous substance such as $\mathrm{KMnO}_{4}$ causes devastating injuries and long-term morbidity among children of Limpopo Province and the country as a whole. Its benefits do not outweigh its dangerous effects. Failure to store the chemical substance in child-proof containers over many years is a clear indication of lack of insight related to its effects. Community educational programmes regarding the dangers of $\mathrm{KMnO}_{4}$ and its storage are needed. Most importantly, it is imperative for the Department of Health to red flag this substance and make it illegal to sell it over the counter, if not ban it completely.

\section{Declaration. None.}

Acknowledgements. The authors would like to thank their institutions for support.

Author contributions. NM initiated the concept and drafted the manuscript. EM read, edited, supervised and approved the manuscript. Funding. None.

Conflicts of interest. None.

1. Street RA, Kabera GM, Connolly C. Ethnopharmacological use of potassium permanganate in South African traditional medicine. S Afr Med J 2018;108(3):187-189. https://doi.org/10.7196/samj.2018. v108i3.12606

2. Holcomb GW III, Murphy JP, St Peter SD, editors. Holcomb and Ashcraft's Pediatric Surgery. 7th ed. New York: Elsevier, 2020

3. Kirsh MM, Ritter F. Caustic ingestion and subsequent damage to the oropharyngeal and digestive passages. Ann Thoracic Surg 1976;21(1):74-82. https://doi.org/10.1016/s0003-4975(10)64894-1

Harris VI. Pyloric stenosis: An unusual complication of alkaline corrosive poisoning. Am J Roentgenol 1968:104(3):594-597. hasis: An unusual complication of all

De Lusong MA, Timbol AB, Tuazon DJ. Management of esophageal caustic injury. World J Gastrointest Pharmacol Ther 2017;8(2):90-98. https://doi.org/10.4292\%2Fwigpt.v8.i2.90

Accepted 10 September 2021. 